\title{
Laparoscopic common bile duct exploration and antegrade biliary stenting: Leaving behind the Kehr tube
}

\author{
Darío Martínez-Baena, Pablo Parra-Membríves, Daniel Díaz-Gómez and José Manuel Lorente-Herce \\ Hepato-Bilio-Pancreatic Surgery Unit. Department of General and Digestive Surgery. Hospital Universitario de Valme. \\ Seville, Spain
}

\begin{abstract}
Introduction: single-stage laparoscopic surgery of cholelithiasis and associated common bile duct stones (CL-CBDS) has shown similar results when compared to laparoscopic cholecystectomy combined with ERCP. Classically, choledochorrhaphy has been protected by a T-tube drain to allow external bypass of bile flow. However, its removal is associated with a significant complication rate. Use of antegrade biliary stents avoids T-tube removal associated morbidity. The aim of this study is to compare the results of choledochorrhaphy plus $T$-tube drainage versus antegrade biliary stenting in our series of laparoscopic common bile duct explorations (LCBDE).

Material and methods: between 2004 and 2011, 75 patients underwent a LCBDE. Choledochorrhaphy was performed following Kehr tube placements in 47 cases and transpapillary biliary stenting was conducted in the remaining 28 patients.

Results: postoperative hospital stay was shorter in the stent group ( $5 \pm 10.26$ days) than in the Kehr group ( $12 \pm 10.6$ days), with a statistically significant difference. There was a greater trend to grade B complications in the stent group (10.7 vs. $4.3 \%)$ and to grade $\mathrm{C}$ complications in the Kehr group (6.4 vs. $3.6 \%$ ). There were 3 cases of residual common bile duct stones in the Kehr group $(6.4 \%)$ and none in the stent group.

Conclusions: antegrade biliary stenting following laparoscopic common bile duct exploration for CL-CBDS is an effective and safe technique that prevents T-tube related morbidity.
\end{abstract}

Key words: Common bile duct stones. Laparoscopic common bile duct exploration. Biliary stenting. T-tube.

Martínez-Baena D, Parra-Membríves P, Díaz-Gómez D, LorenteHerce JM. Laparoscopic common bile duct exploration and antegrade biliary stenting: Leaving behind the Kehr tube. Rev Esp Enferm Dig 2013;105:125-130.

Received: 27-03-2012

Accepted: 18-06-2013

Correspondence: Darío Martínez Baena. Department of General Surgery. Hospital Universitario de Valme. Avenida de Bellavista, s/n. 41014 Seville, Spain

e-mail: dariobaena@gmail.com

\section{INTRODUCTION}

It is estimated that $10-15 \%$ of the patients operated because of gallstone disease have associated choledocholithiasis (1), representing an important potential source of complications (acute pancreatitis, obstructive jaundice, cholangitis).

Treatment of choledocholithiasis has evolved from the first choledocolithotomy described in 1889 by Robert Abbé in New York (2). At the time of open cholecystectomy, the standard practice was single-stage resolution of cholelithiasis with associated common bile duct stones (CLCBDS). It would be necessary to wait almost a century, until 1985, for the first laparoscopic cholecystectomy to be performed (3). Its subsequent generalization and acceptance as the gold standard technique brought a change of scenario in the treatment of common bile duct stones (CBDS). The technical difficulty for single-stage resolution of both, cholelithiasis and CBDS, in the beginnings of laparoscopic surgery favored the generalized use of ERCP before or after laparoscopic cholecystectomy. The next step came as a result of improvements in technology, surgical instruments and surgical technique, resulting in the onset of LCBDE. This technique completes CL-CBDS treatment in a singlestage procedure, providing all the benefits of laparoscopy without the disadvantages associated with ERCP.

Choledochorrhaphy protected by T-tube placement following LCBDE, as well as found in open surgery, still counts as the Achilles heel of this technique. The use of the Kehr tube leads to increased hospital stay due to the general need for progressive clamping and cholangiography performance before complete tube closure is possible, and because of all the potential complications derived from tube removal (biloma, bile peritonitis, etc.) (4).

The aim of this study is to determine if antegrade biliary stenting can substitute the T-tube drainage decreasing complications associated with the use of the latter, but 
maintaining a similar efficacy. Thus, we reviewed the data of the LCBDE that were performed at our institution from 2004 to the present, comparing the patients that received a T-tube drainage with those who underwent antegrade biliary stenting.

\section{MATERIALAND METHODS}

\section{Patients}

According to the management protocol for common bile duct stones of our institution, candidates for single-stage laparoscopic surgery are all patients with a dilated bile duct ( $\geq 10 \mathrm{~mm}$ in diameter) that are fit for surgery and show an acceptable anesthetic risk. All the remaining cases, as well as all patients that had already a cholecystectomy, underwent an ERCP exploration. From March 2004 to February 2011, a total of 85 LCBDE were performed at our unit in patients with CL-CBDS. The conversion rate was $11.76 \%$ (10 patients), establishing a statistically significant relationship to the presence of impacted stones at the papilla (p: 0.04) and total number of stones (p: 0.04). Cases converted to open surgery were excluded from study. In the remaining 75 cases, the operation could be completed by the laparoscopic approach, performing T-tube placements in 47 cases (Kehr group) and antegrade biliary stenting in 28 patients (stent group).

\section{Surgical technique}

The patient is placed in French position, pneumoperitoneum is performed with a supraumbilical Veress needle or a Hasson trocar, followed by the placement of 4 trocars as for laparoscopic cholecystectomy. A $5^{\text {th }}$ trocar is placed at the left flank following choledochotomy. In all patients cholecystectomy was performed at the end of surgery, to allow the gallbladder to be traced up to help to expose the bile duct. Once Calot's triangle was dissected and the cystic duct and artery were identified, transcystic cholangiography was performed if there was a doubt about CBDS presence or about of spontaneous resolution of choledocholithiasis. Supraduodenal longitudinal choledochotomy was performed adjusting its length to the size of the largest stone, followed by choledocolithotomy with saline lavage, Fogarty balloon catheter or Dormia basket passes guided by choledocoscopy. Verification of the absence of residual stones was done in all cases by choledocoscopy or cholangiography.

Closure of the bile duct is performed following the placement of a latex Kehr tube (8 to $14 \mathrm{Fr}$ depending on the diameter of the bile duct) or after antegrade biliary stenting (Flexima $^{\circledast}$ from Boston Scientific Corporation, MA, USA). Since January 2009, we implant a stent as the first option, reserving the use of the Kehr tube for those cases where stent placement is not technically feasible, normally due to impossibility of passing throughout the papilla into the duodenum with the choledocoscope or guide. The choice of diameter $(7-8.5 \mathrm{Fr})$ and length $(5-7 \mathrm{~cm})$ of the stent depends on the characteristics of the bile duct in each case. To ensure transpapillary placement, we use a $0.2 \mathrm{cc}$ Fogarty balloon catheter inserted through the stent itself as a guide. Once the balloon is inflated and secured to the duodenal side of the papilla, the stent is moved distally with a forceps until its distal tab passes through the papilla. The Fogarty balloon catheter is then removed and a new choledocoscopy is performed to ensure transpapillary placement.

Finally, choledochorrhaphy is performed with an interrupted suture of Vicryl 4-0 stitches in the early cases, and since April 2009, with a running suture of the same thread. Water-tightness of the bile duct closure is checked by saline instillation with a cholangiography catheter, and a-Tachosil ${ }^{\circledR}$ patch is then placed over the suture. Finally, cholecystectomy is completed and a subhepatic aspirative drain is left in all cases to drain a potential biliary leak. Biliary leaks were scored according to the recent classification by severity grade published by the International Study Group of Liver Surgery (5).

The postoperative management protocol of the T-tube in our unit involves free flow drainage during the first $72 \mathrm{~h}$, until a trans-Kehr cholangiography is performed. If passage of contrast to the duodenum and absence of residual stones is demonstrated, the tube is clamped $24 \mathrm{~h}$ after cholangiography and the patient is discharged (6). Removal of the tube is performed on an outpatient basis from 30 days after surgery, with clinical observation to rule out complications prior to discharge. Management of patients with a stent is much simpler; outpatient removal of the stent is done by upper endoscopy at 1 month after surgery. Previously, an $\mathrm{X}$-ray image of the abdomen is performed to exclude spontaneous stent elimination.

\section{Statistical analysis}

All data were included prospectively in a specific database (Filemaker Pro ${ }^{\circledR}$ ), and analyzed retrospectively. The SPSS $^{\circledast}$ program was used for statistical analysis. Continuous variables were expressed as the mean \pm standard deviation or as the median when extreme values were observed. The Chi-square test was used for analysis of qualitative variables and the Mann-Whitney test for quantitative variables. Statistical significance was set to $\mathrm{p}<0.05$.

\section{RESULTS}

No significant differences were found between the two groups in their demographic characteristics, symptoms or laboratory parameters (Table I). The moment of diagnosis was mainly in the preoperative period, and the main diagnostic test was cholangio-MRI, followed by ultrasound examination. The average number of extracted stones was 
Table I. Demographic characteristics, symptoms and laboratory parameters

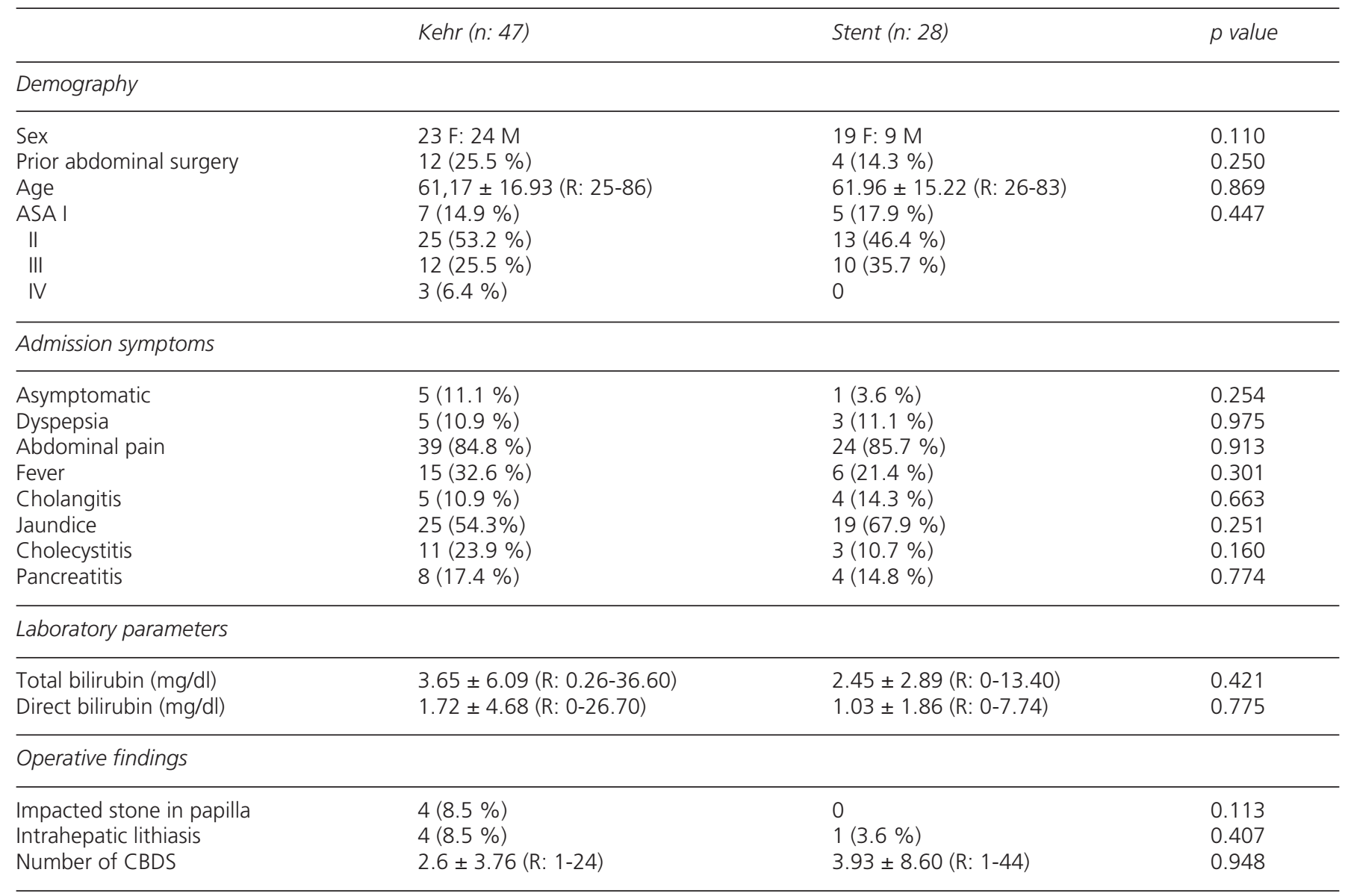

$2.6 \pm 3.76$ (range: $1-24$ ) in the Kehr group and $3.93 \pm 8.60$ (range: $1-44$ ) in the stent group, with no statistically significant difference between the two groups.

Median postoperative hospital stay was $12 \pm 10.6$ days (range: $5-70$ ) in the Kehr group and $5 \pm 10.26$ days in the stent group (range: 1-57), reaching statistical significant differences (p: 0.0005).

We analyzed biliary complications in both groups, including in the Kehr group both those complications related to surgery and complications secondary to T-tube removal (Table II). Comparison of total number of complications or complications stratified by severity revealed no statistical differences between both groups (5). There was a greater trend to grade B complications in the stent group (10.7 vs. $4.3 \%$ ) and to grade $\mathrm{C}$ complications in the Kehr group (6.4 vs. $3.6 \%$ ). We had a single case of iatrogenic injury of the bile duct in the stent group (3.6\%) consisting in a late bile duct scar caused by diathermy. This represents the only case of bile peritonitis in that group.

The rate of readmission was $12.8 \%$ in the Kehr group and $3.6 \%$ in the stent group (p: 0.186). Of the 6 cases of readmission in the Kehr group, 3 were associated to T-tube removal related complications ( 2 cases of bile peritonitis

Table II. Biliary complications ${ }^{\mathrm{a}}$

\begin{tabular}{|c|c|c|c|}
\hline & $K{ }^{b} r^{b}(n: 47)$ & stent (n: 28) & $p$ value \\
\hline \multicolumn{3}{|l|}{ Grade A } & \multirow{7}{*}{0.735} \\
\hline Bile leakage $(<7 d)$ & $2(4.3 \%)$ & $1(3.6 \%)$ & \\
\hline \multicolumn{3}{|l|}{ Grade $B$} & \\
\hline Bile leakage ( $\geq 7 d$ ) & 0 & $3(10.7 \%)$ & \\
\hline Biloma & $2(4.3 \%)$ & 0 & \\
\hline \multicolumn{3}{|l|}{ Grade C } & \\
\hline Bile peritonitis & $3(6.4 \%)$ & $1(3.6 \%)$ & \\
\hline Total & $7(14.89 \%)$ & $5(17.85 \%)$ & 0.706 \\
\hline
\end{tabular}


Table III. General surgical complications

\begin{tabular}{llll}
\hline & Kehr (n: 47) & stent (n: 28) & p value \\
\hline Fever & $4(8,5 \%)$ & $4(14,28 \%)$ & 0,433 \\
Intraabdominal abscess & $2(4,3 \%)$ & $1(3,6 \%)$ & 0,884 \\
Intraabdominal bleeding & $2(4,3 \%)$ & 0 & 0,269 \\
\hline
\end{tabular}

requiring emergency surgery and one case of self-limited biliary leak). The remaining readmissions in the Kehr group were due to 2 cases of residual bile duct stones resolved by ERCP and a case of mild pancreatitis due to distal migration and impaction at the papilla of a branch of the T-tube. The only case of readmission in the stent group was caused by an intra-abdominal collection development that required percutaneous drainage.

The most remarkable non-biliary complications related to surgery were 2 cases of reoperation in the Kehr group due to intra-abdominal bleeding $(4.3 \%)$ (Table III). The overall rate of reoperation was $10.6 \%$ in the Kehr group and $3.6 \%$ in the stent group (p: 0.275).

The rate of residual CBDS (diagnosed within the first year after surgery) was 3 cases in the Kehr group (6.4\%) and none in the stent group, without a statistically significant difference. Bile duct clearance was then achieved by ERCP in all cases; however a post-ERCP severe pancreatitis developed in a patient from the Kehr group causing the only case of death in our series $(2.1 \%)$.

In 11 cases (39.28\%) upper endoscopy was not required for stent removal, since spontaneous elimination was confirmed by a plain X-ray of the abdomen prior to the procedure. Unplanned stent lost was associated to biliary leak only in 2 cases $(7.14 \%)$. The remaining 17 patients underwent endoscopic stent removal without complications.

\section{DISCUSSION}

Treatment of CL-CBDS by single-stage laparoscopic surgery has shown similar results in different studies when compared to laparoscopic cholecystectomy combined with ERCP, with two main advantages over the latter option: it has a better cost-benefit ratio (7-9) and avoids the morbidity and mortality associated to ERCP (10). It should also be taken into account that the success rate of ERCP is approximately $70 \%$ on the first attempt (11), and although it increases in subsequent attempts, most patients will eventually be operated.

In the beginning of LCBDE, the surgical technique was transferred from open choledocolithotomy and choledochorrhaphy protected by a Kehr tube, adapting it to the particularities of laparoscopic approach $(12,13)$. Tissue edema caused by surgical handling of bile duct may block distal passage of bile to duodenum, promoting biliary leakage through the choledochotomy. Use of the T-tube allows an external bypass of bile flow, reducing pressure within the bile duct and ameliorating healing. In addition, it offers the possibility of performing a cholangiography for distal contrast delivery demonstration prior to tube clamping, as well as the opportunity for residual CBDS removal $(14,15)$.

LCBDE offers all the known benefits of the laparoscopic approach when compared to conventional surgery (16), but is clearly limited by the use of the Kehr tube which leads to two essential disadvantages: its management and removal.

Antegrade biliary stenting resulted in a significant reduction of the postoperative hospital stay $(5 \pm 10.26$ days $)$ mainly due to differences in tube management required in the Kehr group, since a follow-up cholangiography and a clamping protocol was necessary before discharge in all cases.

The complications derived from T-tube withdrawal have been widely reported in the literature (17), with an estimated overall morbidity of $15 \%$ both in open and laparoscopic surgery (18), to which the complications derived from surgery itself should be added.

The use of antegrade biliary stents has provided a technical alternative to T-tube placement, ensuring distal passage of bile to the duodenum during the healing process of choledochotomy. Its main advantage consists in avoiding all the disadvantages associated to the Kehr tube. However, biliary stents employment are not absent of specific complications, mainly related to stent migration (19). In spite of this, our data reveal that there were no complications related to stent migration or its endoscopic recovery.

The recent publication of a definition and grading of biliary fistula in liver and bilio-pancreatic surgery by the International Study Group of Liver Surgery (5) has brought standardized criteria about a topic that has traditionally shown as many definitions as study groups possible. In addition, the use of this classification will allow more reliable comparison of results from future clinical studies.

Thus, a biliary fistula is defined as a discharge of drain fluid with a bilirubin concentration at least three times the serum bilirubin concentration on or after the third postoperative day or as the need for radiologic intervention or surgical drainage of biliary collection or bile peritonitis. Concerning severity categorizing, grade A complications are those that do not modify the clinical management of the patient (e.g., fistula exteriorized by drainage lasting < 7 days), grade $\mathrm{B}$ complications are those requiring intervention but not reoperation (e.g., biloma, fistulas exteriorized for 7 or more days) and grade $\mathrm{C}$ biliary complication are those requiring relaparotomy (e. g., bile peritonitis).

In our series, we observed, though without statistical significance, a trend to increased grade B complications in the stent group. Bile leakages were exteriorized throughout the surgical drain and were self-limited in all cases. None of them resulted in bile collections, abscesses or required any additional intervention. Although this leak rate is similar to other reported series (20), it should be noted that of the 3 cases of biliary leakage, 2 of them developed in the first 2 patients from the stent group. We think that the results 
were influenced by our learning curve since we had only 2 cases of leakage in the following 26 patients, (which represents a rate of $7.69 \%$ ), after we modified bile duct closure technique, changing from an interrupted suture to a running suture. Furthermore, the learning curve could also have influenced negatively the presence of residual CBDS in the Kehr group given that 2 of the 3 cases of residual lithiasis were concentrated in the first patients.

We also found a clear trend to an increased rate of both grade $\mathrm{C}$ complications (6.4 vs. $3.6 \%$ ) and the overall reoperation (10.6 vs. $3.6 \%$ ), while without statistical significance in the Kehr group.

It should be noted that we observed a clear tendency to readmission in the Kehr group (12.8vs. $3.6 \%$ in the stent group), with $50 \%$ of readmissions in the Kehr group directly related to complications after tube withdrawal. However, statistical significant differences could not be demonstrated.

We believe the $39.28 \%$ rate of cases of spontaneous migration of the stent is remarkable, since they did not require endoscopic retrieval. It should be considered that if biliary leakage occurred only in 2 of the 11 cases of migration, the presence of the stent might have been unnecessary for healing in the remaining 9 patients. Consequently, primary closure of the bile duct may be safe and feasible, as recommended by the results of different published series (21-23) and may be contemplated as an alternative to consider in the future.

In conclusion, we think that laparoscopic choledochorrhaphy protected by transpapillary biliary stents is an effective and safe technique for single-stage treatment of patients with gallstones and CBDS, by avoiding all the complications derived from management and removal of the Kehr tube, as well as providing a shorter postoperative stay.

Use of a Kehr tube should be reserved for those cases when stent placement technically is not feasible.

\section{REFERENCES}

1. Williams EJ, Green J, Beckingham I. Guidelines on the management of common bile duct stones (CBDS). Gut 2008;57:1004-21.

2. Verbesey JE, Birkett DH. Common bile duct exploration for choledocholithiasis. Surg Clin North Am 2008;88:1315-28, ix.

3. Muhe E. Laparoskopische Cholezystektomie-Spatergebnisse. Langenbecks Arch Chir Suppl Kongressbd 1991:416-23.
4. Dellinger EP, Kirshenbaum G, Weinstein M, Steer M. Determinants of adverse reaction following postoperative T-tube cholangiogram. Ann Surg 1980;191:397-403.

5. Koch M, Garden OJ, Padbury R. Bile leakage after hepatobiliary and pancreatic surgery: A definition and grading of severity by the International Study Group of Liver Surgery. Surgery 2011;149:680-8.

6. Gigot JF. Techniques Chirurgicales Digestif: Chirurgie des voies biliaires. Paris: Masson, 2005.

7. Heili MJ, Wintz NK, Fowler DL. Choledocholithiasis: Endoscopic versus laparoscopic management. Am Surg 1999;65:135-8.

8. Liberman MA, Phillips EH, Carroll BJ. Cost-effective management of complicated choledocholithiasis: Laparoscopic transcystic duct exploration or endoscopic sphincterotomy. J Am Coll Surg 1996;182: 488-94.

9. Rogers SJ, Cello JP, Horn JK. Prospective randomized trial of LC+LCBDE vs. ERCP/S+LC for common bile duct stone disease. Arch Surg 2010;145:28-33.

10. Enochsson L, Swahn F, Arnelo U. Nationwide, population-based data from 11,074 ERCP procedures from the Swedish Registry for Gallstone Surgery and ERCP. Gastrointest Endosc 2010; 72:1175-84, 1184 e1-3.

11. García-Cano Lizcano J, González Martín JA, Pérez Sola A, Morillas Ariño MJ. Success rate of complete extraction of common bile duct stones at first endoscopy attempt. Rev Esp Enferm Dig 2002;94:340-5.

12. Jacobs M, Verdeja JC, Goldstein HS. Laparoscopic choledocholithotomy. J Laparoendosc Surg 1991;1:79-82.

13. Stoker ME, Leveillee RJ, McCann JC, Jr., Maini BS. Laparoscopic common bile duct exploration. J Laparoendosc Surg 1991;1:287-93.

14. Holdsworth RJ, Sadek SA, Ambikar S, Cuschieri A. Dynamics of bile flow through the human choledochal sphincter following exploration of the common bile duct. World J Surg 1989;13:300-4; discussion 305-6.

15. Burhenne HJ. Garland lecture. Percutaneous extraction of retained biliary tract stones: 661 patients. AJR Am J Roentgenol 1980;134: 889-98.

16. Berggren U, Gordh T, Grama D. Laparoscopic versus open cholecystectomy: Hospitalization, sick leave, analgesia and trauma responses. Br J Surg 1994;81:1362-5.

17. Moreaux J. Traditional surgical management of common bile duct stones: A prospective study during a 20-year experience. Am J Surg 1995;169:220-6.

18. Wills VL, Gibson K, Karihaloot C, Jorgensen JO. Complications of biliary T-tubes after choledochotomy. ANZ J Surg 2002;72:177-80.

19. Isla AM, Griniatsos J, Karvounis E, Arbuckle JD. Advantages of laparoscopic stented choledochorrhaphy over T-tube placement. Br J Surg 2004;91:862-6.

20. Tang CN, Tai CK, Ha JP, Tsui KK, Wong DC, Li MK. Antegrade biliary stenting versus T-tube drainage after laparoscopic choledochotomy - A comparative cohort study. Hepatogastroenterology 2006; 53:330-4.

21. Decker G, Borie F, Millat B, Berthou JC, Deleuze A, Drouard F, et al One hundred laparoscopic choledochotomies with primary closure of the common bile duct. Surg Endosc 2003;17:12-8.

22. Jameel M, Darmas B, Baker A. Trend towards primary closure following laparoscopic exploration of the common bile duct. Ann R Coll Surg Engl 2008;90:29-35.

23. Zhang WJ, Xu GF, Wu GZ, Li JM, Dong ZT, Mo XD. Laparoscopic exploration of common bile duct with primary closure versus T-tube: A randomized clinical trial. J Surgical Research 2009; 157: e1-e5. 